\title{
Die Selektion auf Wurfgröße beim Schwein
}

\section{Herrn Professor Dr. Peter Glodek zum 65. Geburtstag gewidmet}

\begin{abstract}
Summary
Title of the paper: Selection for litter size in swine

The present paper deals with the estimation of genetic parameter for litter size of two purebred lines 03 and 04 in order to choose the optimal model for variance component estimation. Data from nucleus and multiplier farms from 1989 to 1997 of a North-German breeding company were observed. 9,957 litter records from 3,423 sows of line 03 and 5,045 litter records from 2,009 sows of line 04 were collected. The 404 sires of line 03 had an average of 8.5 daughters and the 285 sires of line 04 had an average of 7,0 daughters. Litter size were different between both lines, $9.6(3.2)$ and 10.1 (3.4) for line 03 and 04 , respectively.

Genetic correlations between litters of parity 1, 2 and 3 were $r_{g} \geq 0,81$ on high level. Also, in bivariate analysis high correlations $r_{g} \geq 0,88$ were estimated for both lines. This model takes the maternal effect for the first parity and the permanent environment in subsequent parities into account. Due to the results a repeatability model may be appropiate for breeding values of litter size. Estimation of genotype by environment interaction showed different heritabilities between farms. Genetic corrlation was on moderate level $\left(r_{g}=0,61\right)$ between two farms for line 04 , other estimates ranged between $r_{g}=0,73$ and 0,97 .
\end{abstract}

Key Words: swine, litter size, repeatability model, genetic correlation

\section{Zusammenfassung}

In der vorliegenden Untersuchung wurden genetische Parameter für die Wurfgröße von zwei Reinzuchtlinien 03 und 04 geschätzt, um das optimale Modell für die Varianzkomponentenschätzung auszuwählen. Die Datengrundlage bilden Produktionsaufzeichnungen von Nukleus- und Vermehrerbetrieben eines norddeutschen Zuchtunternehmens, die in einem Zeitraum von 1989 bis 1997 erfaßt wurden. Dabei standen insgesamt 9.957 Wurfleistungen von 3.423 Sauen der Linie 03 und 5.045 Wurfleistungen von 2.009 Sauen der Linie 04 zur Verfügung. Die 404 Eber der Linie 03 haben im Mittel 8,5 Töchter während 285 Eber der Linie 04 im Mittel 7,0 Töchter aufweisen. Die beiden Linien zeigen unterschiedliche Wurfgrößen mit 9,6 $(3,2)$ lebend geborenen Ferkeln (LGF) für Linie 03 und 10,1 (3,4) LGF für Linie 04.

Die genetischen Korrelationen zwischen den Würfen 1, 2 und 3 liegen mit $r_{g} \geq 0,81$ auf einem hohen Niveau. Auch mit einem bivariaten Modell werden hohe Korrelationen zwischen dem ersten und den Folgewürfen von $r_{g} \geq 0,88$ für beide Linien geschätzt. Die Ergebnisse erlauben die Anwendung eines Wiederholbarkeitsmodells für die Zuchtwertschätzung der Wurfgröße. Die Schätzung von Genotyp-Umwelt-Interaktionen zeigt unterschiedliche Heritabilitäten für die jeweiligen Betriebe. Die genetische Korrelation wird nur zwischen zwei Nukleusbetrieben auf einem mittleren Niveau $\left(r_{8}=0,61\right)$ angegeben, sonst liegen sie zwischen $r_{8}=0,73$ bis 0,97 .

Schlüsselwörter: Schwein, Wurfgröße, Wiederholbarkeitsmodell, genetische Korrelation

- Die Untersuchungen wurden mit dankenswerter finanzieller Unterstatzung der Deutschen Forschungsgemeinschaf (DFG) angefertigt. 


\section{Einleitung und Literatur}

Die Wurfgröße ist ein bedeutendes funktionales Merkmal in der Schweinezucht. Sie wird durch die Anzahl lebend geborene Ferkel erfaßt und in Zuchtprogrammen durch gezielte Selektion in spezialisierten Mutterlinien weiterentwickelt. Die Heritabilität liegt, wie für Merkmale der Fruchtbarkeit und Vitalität üblich, mit $h^{2}=0,10$ (HALEY et al., 1988) auf einem niedrigen Niveau.

In der Literatur werden verschiedene Modelle zum Schätzen von Varianzkomponenten und Zuchtwerten für die Wurfgröße angewandt. In Tabelle 1 ist eine Zusammenstellung der systematischen Einflußfaktoren aufgeführt, die in Modellen zur Schätzung der genetischen Parameter für Wurfgröße berücksichtigt werden.

HERMESCH (1996) und FISCHER (1998) wenden modifizierte Wiederholbarkeitsmodelle an, mit denen sowohl maternale additiv genetische Effekte $\left(\mathrm{m}^{2}\right)$ für den ersten Wurf als auch permanente Umwelteffekte für die wiederholten Wurfleistungen ab dem zweiten Wurf geschätzt werden können.

Tabelle 1

Systematische und zufällige Einflußfaktoren in genetischen Schätzmodellen für Wurfgröße (Systematic and random factors in genetic models for litter size)

\begin{tabular}{lll}
\hline Fixer Effekt & Kovariable & Zufalliger Effekt \\
\hline Rasse der Sau & Erstferkelalter & Tier-Mehrmerkmalsmodell \\
Herkunftsbetrieb & Zwischenwurfzeit & Tier-Wiederholbarkeitsmodell \\
Paarungsart & Alter der Sau beim Wurf & Paternaler und maternaler Effekt \\
Linie des Belegeebers & Gewicht der Sau & Vater des Wurfes \\
Alter bei Belegung & & Herkunftswurf \\
Herde-Jahr-Saison, Wurfmonat & Resteffekt \\
Wurfnummer, Wurfnummernklasse & \\
\hline HALEY und LEE (1992); SEE et al. (1993); IRGANG et al. (1994); ESTANY und SøRENSEN (1995); ROHE \\
$\begin{array}{l}\text { und KENNEDY (1995); HERMESCH (1996); ALFONSO et al. (1997); BÖSCH et al. (1997); FISCHER } \\
\text { (1998); THOLEN et al. (1998) }\end{array}$ \\
\hline
\end{tabular}

Verschiedene Autoren schätzen in ihren Untersuchungen eine negative Korrelation zwischen maternalen additiv genetischen Effekten und direkten genetischen Effekten für die Wurfgröße beim ersten Wurf von $\mathrm{r}_{\mathrm{am}}=-0,07$ bis $-0,99$ (JØRGENSEN, 1989; SOUTHWOOD und KENNEDY, 1990; LOOFT, 1992; FERRAZ und JOHNSON, 1993; RÖHE und KENNEDY, 1993; FISCHER, 1998). Die Heritabilitäten weisen in diesen Untersuchungen im Mittel $h^{2}{ }_{m}=0,04$ in einem Bereich von 0,02 bis 0,08 auf. Aufgrund des niedrigen Erblichkeitsgrades und des allgemein praktizierten Wurfausgleichs wird dieser genetische Effekt in der Varianzanalyse vernachlässigt.

Zahlreiche Autoren berücksichtigen die permanente Umweltvarianz (pu) in ihren Untersuchungen. Der phänotypische Anteil der permanenten Umweltvarianz wird in der Literatur zwischen $\mathrm{pu}=0,04$ und 0,10 angegeben und sind populationsspezifisch (SØRENSEN et al., 1990; ESTANY und SØRENSEN, 1995; TÖLLE und TRAPPMANN, 1995; FREY et al., 1995; TÄUBERT et al., 1997; FISCHER, 1998). Die Autoren vernachlässigen bei dieser Vorgehensweise, daß die genetische Korrelation zwischen einzelnen Würfen niedriger als 1 und die Heritabilitäten der aufeinanderfolgen- 
den Würfe nicht selten auf unterschiedlichem Niveau liegen. Der Selektionsfortschritt kann dadurch mit einem Wiederholbarkeitsmodell überschätzt werden.

In einer Zusammenstellung von sieben Literaturquellen (IRGANG et al., 1994; RÖHE und KENNEDY, 1995; HERMESCH, 1996; THOLEN et al., 1996; ALFONSO et al., 1997; FISCHER, 1998 und TÄUBERT, 1998) beträgt der Median der Schätzwerte für die genetischen Korrelationen zwischen dem ersten und zweiten Wurf $\mathrm{r}_{\mathrm{g} 1: 2}=0,67\left(\mathrm{r}_{\mathrm{g} 1: 2}=\right.$ $0,37$ bis 0,92$)$, zwischen dem ersten und dritten Wurf $r_{g 1: 3}=0,77\left(r_{g 1: 3}=0,47\right.$ bis 1,0$)$ und zwischen den Wurfnummern 2 und $3 r_{g 2: 3}=0,96\left(r_{g 2: 3}=0,60-1,0\right)$. FISCHER (1998) schätzt die genetische Korrelationen zwischen den Würfen 1 und 2 bis 10 auf $r_{g 1: 2+}=$ 0,88 und 0,82 für Deutsches Edelschwein und Deutsche Landrasse.

Die Heritabilitäten für die einzelnen Wurfnummern werden uneinheitlich angegeben und sind wie die genetischen Korrelationen linien- bzw. populationsspezifisch. Sie werden im zweiten und in einigen Populationen auch für den dritten Wurf (RÖHE und KENNEDY, 1995; IRGANG et al., 1994 und ALFONSO et al., 1997) höher als für den ersten Wurf geschätzt. IRGANG et al. (1994), RÖHE und KENNEDY (1995), HERMESCH (1996) und FISCHER (1998) folgern aus ihren Ergebnissen, daß die Zuchtwerte für die einzelnen Wurfleistungen als getrennte Merkmale betrachtet werden sollten.

Wird ein Wiederholbarkeitsmodell angewandt, können die Niveauunterschiede von wiederholten Leistungen der Wurfnummern 3 bis 5 sowie der Würfe $\geq 6$ durch einen fixen Effekt modelliert werden (ESTANY und SØRENSEN, 1995; ALFONSO et al., 1997; BÖSCH et al., 1997). Verschiedene Autoren führen zusätzlich eine Korrektur des Alters innerhalb jeder Wurfnummer durch (HERMESCH, 1996; BRISBANE et al., 1998; HOFER et al., 1998; TRIBOUT et al., 1998). Damit fließt die Beziehung zwischen Alter und Wurfgröße ein, die insbesondere beim ersten Wurf besteht.

\section{Erstferkelalter}

BUNTER (1997) schätzt für das Erstferkelalter eine Heritabilität von $\mathrm{h}^{2}=0,11$ und die genetische Korrelation zur Wurfgröße mit annähernd Null. Das Erstferkelalter wird wesentlich vom Pubertätsalter und dem Abstand zwischen erstem Östrus und erfolgreicher Konzeption bestimmt. Die Heritabilität für das Alter bei Geschlechtsreife wird in verschiedenen Untersuchungen auf $h^{2}=0,10$ bis 0,34 mit einem Median von $\mathrm{h}^{2}=0,24$ geschätzt (HALEY et al., 1988; LAMBERSON et al., 1991; BIDANEL et al., 1992; RYDHMER et al., 1994), jedoch liegt die genetische Korrelation zur Wurfgröße nahe Null (HALEY et al., 1988).

\section{Zwischenwurfzeit}

Die Zwischenwurfzeit (ZWZ) hängt von der Dauer der Tragezeit, der Laktationslänge und dem Intervall zwischen Absetzen und erfolgreicher Belegung ab. Die Dauer der Trächtigkeit weist eine geringe Variation auf $(s=1,5$ Tage) und hat zur Wurfgröße eine niedrige Beziehung von $r_{g}=-0,16$ bzw. $r_{g}=-0,23$ (OMTVEDT et al., 1965; WILLEKE und RICHTER, 1980). Die ZWZ wird in der Regel als Kovariable in Schätzmodellen für Wurfleistung berücksichtigt. Sie weist allerdings nur zwischen dem ersten und zweiten Wurf einen signifikanten Einfluß auf(TÄUBERT, 1998). 


\section{Herde-Jahres-Saison Effekt}

Der Herde-Jahres-Saison Effekt spiegelt die individuelle Betriebsumwelt, das Management und den Gesundheitsstatus eines Betriebes wider, das sowohl bedingt durch klimatische Gegebenheiten innerhalb eines Jahres wie auch zwischen den Jahren variieren kann. Insbesondere lösen hohe Temperaturen Streß aus, der zu einer verzögerten Brunst, verringerter Konzeptionsrate, erhöhter Umrauscherquote und erhöhter embryonalen Sterblichkeit führen kann (GRANDJOT et al., 1997). Herde-Jahres-Saison oder Herde-Jahres Effekte können als zufällige oder fixe Effekte im Modell berücksichtigt werden. Eine Auswirkung der unterschiedlichen Modellformulierung ist zu erwarten, wenn die Genotypen nicht zufällig über die Betriebe verteilt sind (FREY et al., 1995).

\section{Paarungsart und Belegeeber}

In zahlreichen Untersuchungen wird eine Überlegenheit des Natursprungs über die künstliche Besamung von 0,2 bis 0,5 Ferkeln festgestellt (WILLEKE und RICHTER, 1980; SØRENSEN, 1990; FREY et al., 1994).

WILLEKE und RICHTER (1980) zeigen, daß der Faktor Eber einen hochsignifikanten Einfluß auf die Wurfgröße hat. Hingegen schätzen SEE et al. (1993) den Anteil des Wurfvaters an der Gesamtvarianz auf lediglich 1 bis $3 \%$ für drei verschiedene Rassen. Der Belegeeber wird von einigen Autoren als fixer Effekt bei der Varianzkomponentenschätzung berücksichtigt (ANDERSEN, 1998; BRISBANE et al., 1998; TRIBOUT et al., 1998; WOLF et al., 1998).

\section{Genotyp-Umwelt-Interaktionen}

Eine Interaktion zwischen Genotyp und Umwelt besteht, wenn eine bestimmte Umweltdifferenz zu einem unterschiedlichen Effekt bei verschiedenen Genotypen führt, so daß sogar Rangfolgeunterschiede der Genotypen auftreten können, wenn sie in unterschiedlichen Umwelten gemessen werden FALCONER (1996). MERKS (1988) leitet aus seinen Untersuchungen bei Mastschweinen ab, daß Genotyp-Umwelt Interaktionen hauptsächlich auf Wechselwirkungen zwischen dem genetischen Potential und dem Management auf den Betrieben beruhen. Die genetische Korrelation zwischen Umwelten stellt nach FALCONER (1996) ein Mittel zur Quantifizierung der Interaktionen fưr die Schätzung des Selektionserfolges dar. Eine niedrige genetische Korrelation weist auf Genotyp-Umwelt-Interaktionen hin, die zu einer unterschiedlichen Rangfolge der in differenzierten Umwelten geprüften Eber führen kann. Genauso kann die absolute oder relative Höhe der Varianzen zwischen den Betriebsumwelten unterschiedlich sein. Über eine reduzierte Genauigkeit der Zuchtwertschätzung wirken sich Interaktionen negativ auf den Zuchtfortschritt aus. Das Ausmaß hängt von der Höhe der Korrelation zwischen den Umwelten ab.

Aus einer Literaturzusammenstellung schließt VON FELDE (1996), daß nur wenige Arbeiten das Auftreten von Genotyp-Umwelt-Interaktionen bestätigen und eine gute Übereinstimmung zwischen den meisten Leistungsprüfvarianten für Produktionsmerkmale beim Schwein besteht.

Der jeweilige Median der genetischen Korrelationen für tägliche Zunahme, Lebens- 
tagszunahme, Rückenspeckdicke und Muskelfleischanteil wird mit $r_{g}=0,70,0,88,0,82$ und 0,70 , sowie mit $r_{g}=0,38,0,23$ und 0,43 für Futterverwertung, Futteraufnahme und Restfutteraufnahme angegeben. Schätzungen der Genotyp-Umwelt-Interaktionen für Fruchtbarkeitsmerkmale sind bisher nicht verfügbar.

\section{Ziel der Arbeit}

Das Ziel dieser Arbeit ist die Schätzung der genetischen Parameter für die Wurfleistung von aufeinanderfolgenden Würfen von zwei Mutterlinien an einem praktischen Datensatz. Dabei werden verschiedene Modellvarianten hinsichtlich ihrer Eignung als optimale Schätzmethode miteinander verglichen. In einer Auswertung an einem Teildatensatz werden genetische Parameter für unterschiedliche Paarungsarten geschätzt. Weiterhin wird untersucht, ob Genotyp-Umwelt Interaktionen zwischen Nukleus- und Vermehrerbetrieben wirken.

\section{Material und Methoden}

\section{Datenstruktur}

Die Datengrundlage für die Untersuchungen bilden Produktionsaufzeichnungen von Nukleus- und Vermehrerbetrieben eines norddeutschen Zuchtunternehmens. Die Wurfleistungen, als Merkmal lebend geborene Ferkel erfaßt, werden von zwei Reinzuchtlinien 03 und 04 aus den Jahren 1989 bis 1997 berücksichtigt. Die Varianzkomponentenschätzungen werden mit den Programmpaketen VCE4 von GROENEVELD (1998) und MTDFREML. von BOLDMAN et al. (1993) durchgeführt. Beide Mutterlinien werden auf Nukleusbetrieben züchterisch weiterentwickelt. Die Töchter werden an nachgelagerte Vermehrerbetriebe geliefert.

Für die genetische Analyse stehen 9.957 Würfe von 3.423 Sauen der Linie 03 zur Verfügung. Von Linie 04 gehen 5.045 Würfe von 2.009 Sauen in die genetische Analyse ein, wie Tabelle 2 zeigt. Bei Linie 03 stehen annähernd 25 Würfe je Eber und bei Linie 0418 Würfe je Eber zur Verfügung. Die Eber wurden im Mittel auf 1,6 Betrieben (1-3 Betriebe) eingesetzt, so daß die genetische Verknüpfung der Betriebe gewährleistet ist. Gerade bei Merkmalen mit niedriger Erblichkeit wie die Wurfgröße sind Halbgeschwisterinformationen wichtig. Sie werden durch den überbetrieblichen KB-Einsatz der Eber erzielt.

Tabelle 2

Daxenstruktur der Reinzuchtlinien 03 und 04 (Structure of data for purebred lines 03 and 04)

\begin{tabular}{l|c|c}
\hline Linie & 03 & 04 \\
\hline Würfe & 9.957 & 5.045 \\
Sauen & 3.423 & 2.009 \\
Würfe je Sau & 2,9 & 2,5 \\
Väter & 404 & 285 \\
Sauen je Vater & 8,5 & 7,0 \\
Würfe je Vater & 24,6 & 17,7 \\
Müitrer & 1.791 & 1.060 \\
Sauten je Murter & 1,9 & 1,9 \\
\hline
\end{tabular}




\section{Fruchtbarkeitsdaten}

Das Erstferkelalter wurde in Grenzen von 294 bis 450 Tagen und die Zwischenwurfzeit im Bereich von 120 bis 250 Tagen berücksichtigt. Die Saison wurde in vier Klassen eingeteilt: Dezember bis Februar, März bis Mai, Juni bis August und September bis November. Weitere Einflußfaktoren sind die Paarungsart, künstliche Besamung oder Natursprung, die Wurfnummern 1 bis 9, von denen die Wurfnummern 3 bis 5 und 6 bis 9 jeweils gruppiert wurden und der Herde-Jahres-Saison Effekt. Die folgende Tabelle 3 zeigt Mittelwerte für die Wurfgröße der beiden Linien und deren Einflußfakroren.

Tabelle 3

Mittelwerte für Wurfleistungen und deren Einflußfaktoren der Linien 03 und 04 (Means for litter performance and their factors of influence for line 03 and 04)

\begin{tabular}{llcc}
\hline & & Linie 03 & Linie 04 \\
\hline LGF & $\overline{\mathrm{x}}(\mathrm{s})$ & $9,64(3,17)$ & $10,08(3,39)$ \\
EFA & Tage (s) & $339(22)$ & $337(21)$ \\
ZWZ & Tage (s) & $147(8)$ & $148(15)$ \\
KB & $(\%)$ & 84 & 97 \\
Wurfiur. & $\varnothing$ & 2,9 & 2,5 \\
HJS (min.) & & 26 & 20 \\
\hline
\end{tabular}

LGF $=$ Lebend geborenc Fericel, EFA $=$ Erstierkelaiter, $Z W Z=Z$ wischenwurfzeti, KB= Konstliche Besamung, HJS $=$ Herde-Jahr-Saison (minimale Klassenbesezzung)

Die Linie 04 weist mit 10,08 LGF größere Würfe als Linie 03 mit 9,64 LGF auf. Eine mögliche Ursache ist darin zu sehen, daß Linie 04 in der Vergangenheit stärker auf Wurfgröße selektiert worden ist, wie Untersuchungen von LOOFT (1992) zeigen. Der KB-Anteil liegt bei der Linie 03 mit $84 \%$ niedriger als bei Linie 04 mit $97 \%$. Die Interaktion zwischen Herde, Jahr und Saison zeigt eine ausreichende minimale Subzellenbesetzung für die Beruicksichtigung als fixer Effekt. Beim Erstferkelalter und der Zwischenwurfzeit bestehen mit 339 bzw. 337 Tagen und 147 bzw. 148 Tagen kaum Unterschiede zwischen den berrachteten Linien. Der Abstand zwischen dem ersten und zweiten Wurf ist größer als zwischen den späteren Würfen.

Die Signifikanzniveaus der fixen und zufälligen Einflußfaktoren auf die Wurfgröße (LGF) wurden mit der GLM-Prozedur in SAS (1995) getestet. Sowohl die Wurfnummer als auch die Herde-Jahres-Saison Effekte (HJS) sind bei allen Linien sehr hoch signifikant. Die Interaktion Herde*Paarungsart ist bei Linie 04 nicht signifikant, was durch die Datenstruktur erklärt werden kann.

Tabelle 4

Signifikanzniveaus der fixen und zufälligen Einflußfaktoren auf die Wurfgröße (LGF) der Linien 03 und 04 (Level of significance for fixed and random factors for litter size, NBA)

\begin{tabular}{|c|c|c|}
\hline & 03 & 04 \\
\hline Herde-Jainr-Saison & *क⿻ा一ा & 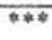 \\
\hline Wurfnummerngruppe & $\omega *$ & $* * *$ \\
\hline Herde*Paanungsart & $* *$ & n.s. \\
\hline Erstferkelalter & $* * *$ & $* *$ \\
\hline Zwischenwurfzeit & * & * \\
\hline$r^{2} \%$ & 8,0 & 7,4 \\
\hline
\end{tabular}

1) $p>0,05=$ nicht signifikant n.s.; $0,01>p \leq 0,0 S=$ signifikant ${ }^{*} ; 0,001>p \leq 0,01=$ hoch signifikant **; $p<0,001=$ sehr hoch signifikant $\Delta v 0$ 
Das Erstferkelaiter (EFA) wird in vier Klassen eingeteilt: 294-318 Tage, 319-338 Tage, 339-358 Tage und 359-450 Tage. Die Zwischenwurfzeit (ZWZ) wird in drei Klassen eingeteilt: 120-138 Tage, 139-158 Tage und 159-250 Tage. Die erste Gruppe umfaßt die Sauen, die eine außergewöhnlich niedrige Zwischenwurfzeit aufweisen. In der letzten Gruppe werden sogenannte Problemsauen zusammengefaßt, die Schwierigkeiten haben, erneut tragend zu werden. Die Berücksichtigung von EFA und ZWZ als fixen Effekt oder als Kovariable zeigt keine Unterschiede beim Signifikanztest. Die Bestimmtheitsmaße $\left(\mathrm{r}^{2}\right)$ liegen mit $8 \%$ bzw. $7 \%$ auf niedrigem Niveau. In der Abbildung werden für die Linien 03 und 04 Least Square Means der Wurfgröße gezeigt. Die berücksichtigten Einflußfaktoren sind neben der Wurfnummernklasse, die sich aus Wurfnummer und EFA-Klasse bzw. ZWZ-Klasse zusammensetzt, die Paarungsart und die Herde-Jahres-Saison Effekte.

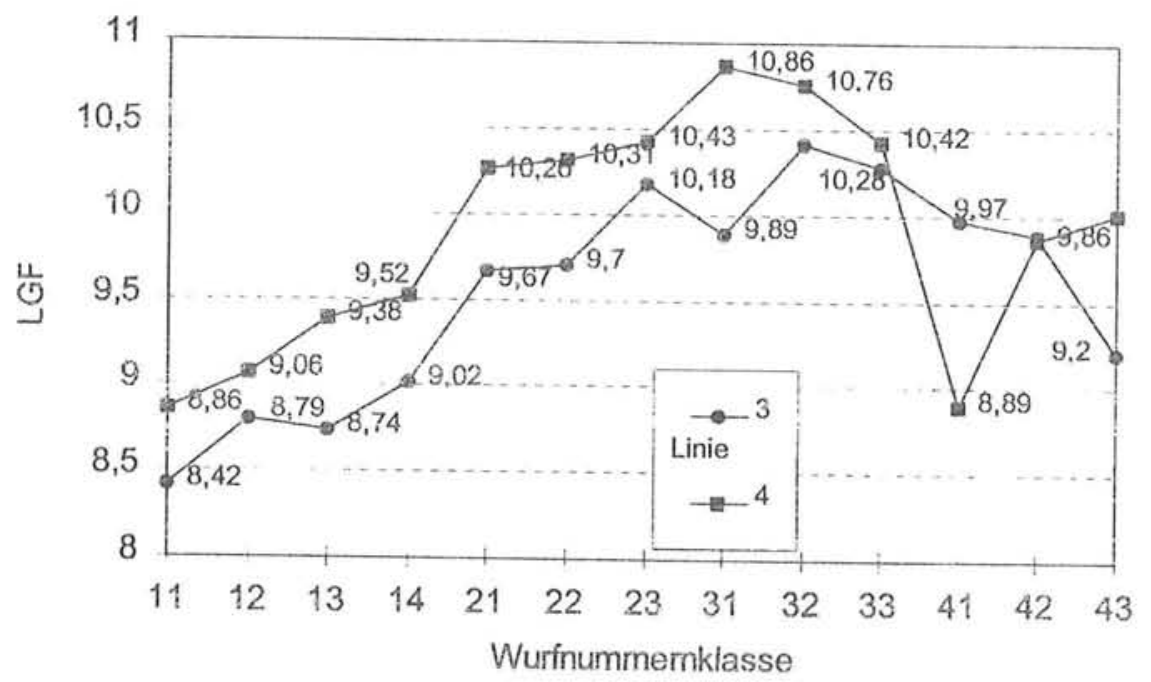

Abb.: Least Square Means für den Verlauf der Wurfgröße (LGF) in Abhängigkeit von der Wurfnummer, dem Erstferkelalter und der Zwischenwurfzeit (Wurfnummernklasse) für die Linien 03 und 04 (Least Square Means for litter size, NBA, dependent on parity, first farrowing age and breeding intervall for line 03 and 04 )

Die Sauen mit einem hohen Erstferkelalter bei Wurfnummer 1 haben in der Regel gröBere Würfe. Dieser nahezu lineare Trend setzt sich auch in abgeschwächter Weise noch beim zweiten Wurf mit zunehmender Zwischenwurfzeit fort. Die Ursache liegt vermutilich darin, daß die Sau in diesem Stadium noch nicht ausgewachsen ist. Die Fähigkeit, große Würfe prë- und postnatal zu versorgen, wird erst mit zunehmendem Alter voll ausgebildet. Bis zur Wurfnummerngruppe $3(31,32,33)$, die sich aus den Wurfmummern 3 bis 5 und den drei Klassen der Zwischenwurfzeit zusammensetzt, steigt die Wurfgröße an und sinkt bei Wurfnummer 6 wieder ab. Durch die Einteilung der Zwischenwurfzeit in drei Klassen kann gezeigt werden, daß eine mittlere Zwischenwurfzeit optimal für die Fruchtbarkeit ist. 


\section{Modelle für die Varianzkomponentenschätzung}

Die genetischen Parameter zwischen den Würfen 1 bis 3 werden für jede Linie getrennt nach Modell [1] geschätzt.

$$
\mathbf{y}_{\mathrm{ijkl}}=\mu+\mathrm{HJS}_{\mathrm{il}}+\mathbf{P A}_{\mathrm{jl}}+\mathbf{a}_{\mathrm{ijkl}}+\mathbf{b}^{*}\left(\mathrm{x}_{\mathrm{ijk} 1}\right)+\mathbf{e}_{\mathrm{ijk}}
$$

$\begin{array}{ll}\mathrm{y}_{\mathrm{ijkk}} & \text { Beobachtung des k-ten Tieres im 1-ten Wurf } \\ \mathrm{HJS}_{\mathrm{il}} & \text { fixer Effekt der i-ten Herde-Jahr-Saison Klasse } \\ \mathrm{PA}_{\mathrm{j} !} & \text { fixer Effekt der j-ten Paarungsart } \\ \mathrm{a}_{\mathrm{ijkl}} & \text { zufälliger additiv genetischer Effekt des k-ten Tieres } \\ \mathrm{b}^{*}\left(\mathrm{x}_{\mathrm{ijk})}\right) & \text { lineare Regression der Beobachtung auf das Erstferkelalter (1. Wurf) } \\ & \text { und der Zwischenwurfzeit (2. und 3. Wurf) } \mathrm{x}_{\mathrm{ijkl}} \\ \mathrm{e}_{\mathrm{ijkl}} & \text { zufälliger Rest- (Umweit-) Effekt. }\end{array}$

Mit dem folgenden Modell [2] werden genetische Korrelationen zwischen dem ersten Wurf́ und den höheren Würfen geschätzt. Damit werden ein Mehrmerkmalsmodell und ein Wiederholbarkeitsmodell kombiniert. Diese Differenzierung erlaubt das Schätzen von maternalen additiv genetischen Effekten für den ersten Wurf und permanenten Umwelteffekten für die höheren Wurfnummern. Dabei kann eine Steigerung der Heritabilität durch eine Verringerung der temporären Umwelteinflüsse erwartet werden (LOOFT, 1992). Mit diesem Modell werden das Erstferkelalter in vier und die Zwischenwurfzeit innerhalb der Wurfnummerngruppe in drei fixen Effektklassen zusammen mit der Wurfnummernklasse berücksichtigt.

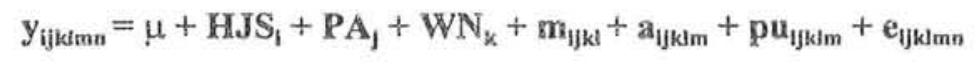

$\begin{array}{ll}\text {HJS }_{\mathrm{i}} & \text { fixer Effekt der i-ten Herde-Jahr-Saison Klasse } \\ \mathrm{PA}_{\mathrm{j}} & \text { fixer Effekt der j-ten Paarungsart } \\ \mathrm{WN}_{\mathrm{k}} & \text { fixer Effekt der k-ten Wurfnummernklasse } \\ \mathrm{m}_{\mathrm{ijk}} & \text { zufälliger maternaler genetischer Effekt der 1-ten Mutter } \\ \mathrm{a}_{\mathrm{ijklm}} & \text { zufälliger additiv genetischer Effekt des m-ten Tieres } \\ \mathrm{pu}_{\mathrm{ijklm}} & \text { zufälliger permanenter Umwelteffekt des m-ten Tieres } \\ \mathrm{e}_{\mathrm{ijkmmn}} & \text { zufälliger Rest- (Umwelt-) Effekt. }\end{array}$

Mit dem Wiederholbarkeitsmodell [3] werden genetische Korrelationen zwischen Betrieben der Reinzuchtstufe furr die Wurfleistung geschätzt.

$$
y_{i j k i m n}=\mu+J S_{i}+P A_{j}+W N_{k}+L_{1(03,04 / N I I / v I I)}+a_{i j k l m}+p_{i j k l m}+e_{i j k l m n}
$$

Von Betrieb NII wird zusätzlich der fixe Effekt der Linie des Belegeebers $\left(\mathrm{LE}_{1\left(03 / 04 \_\mathrm{NII} / \mathrm{VII}\right)}\right)$ berücksichtigt, da auf diesem Betrieb auch reziproke Anpaarungen durchgeführt werden. Bei den Betrieben NI und VI ist die Linie des Belegeebers mit dem Betrieb confounded.

Ergebnisse und Diskussion

Genetische Parameter fur aufeinanderfolgende Wurfleistungen

Anhand von Modellgleichung [1] werden genetische Parameter für die Würfe 1 bis 3 
mit dem Programmpaket MTDFREML (BOLDMAN et al., 1993) geschätzt (Tab. 5). Beim dritten Wurf von Linie 03 wird die höchste Heritabilität der Wurfgröße mit $\mathrm{h}^{2}=$ 0,17 geschätzt. Das kann damit begründet werden, daß gegenüber früheren Würfen das genetische Leistungsvermögen am besten ausgeprägt ist. Im Gegensatz dazu wird bei Linie 04 die höchste Heritabilität für den ersten Wurf geschätzt $\left(h^{2}=0,17\right)$. Hier werden linienspezifische Unterschiede deutlich, die auch TÄUBERT (1998) in seinen Untersuchungen an Mutterlinien bestätigt.

Die höchste phänotypische Varianz wird für beide Linien beim zweiten Wurf festgestellt. Die Kovarianzen und genetischen Korrelationen zwischen den Würfen nehmen bei Linie 03 mit steigender Wurfnummer zu. Die genetischen Korrelationen zwischen dem ersten und zweiten Wurf zeigen mit $r_{g}=0,81$ und 0,98 (Linie 03 bzw. 04) im Vergleich zur Literatur eine hohe genetische Beziehung zwischen den Würfen (Tab. 5). Zwischen dem ersten und dritten Wurf bzw. zweiten und dritten Wurf werden ebenfalls hohe genetische Korrelationen von $r_{g}=0,87$ und 0,99 für Linie 03 bzw. $r_{g}=0,89$ und 0,96 für Linie 04 geschätzt.

Tabelle 5

Genetische Parameter für lebend geborene Ferkel der Wurfnummern 1 bis 3 geschätzt für die Linien 03 und 04 (Genetic parameter for live born piglets of parity 1 to 3 for the lines 03 and 04)

\begin{tabular}{|c|c|c|c|c|c|c|}
\hline \multirow[b]{2}{*}{ Wurfnr. } & \multicolumn{3}{|c|}{ Linie 03} & \multicolumn{3}{|c|}{ Linie 04} \\
\hline & 1 & 2 & 3 & 1 & 2 & 3 \\
\hline$\sigma_{a-1}^{2}$ & 0,78 & & & 1,59 & & \\
\hline $\operatorname{Cov}_{\mathrm{a}} / \sigma_{\mathrm{a}, 2}^{2}$ & 0,70 & 0,95 & & 1,50 & 1,46 & \\
\hline $\operatorname{Cov}_{\mathrm{a}} / \sigma_{\mathrm{a}_{3} 3}^{2}$ & 1,00 & 1,26 & 1,68 & 1,30 & 1,34 & 1,34 \\
\hline$\sigma^{2}=$ & 8,25 & 9,42 & 9,03 & 7,99 & 10,46 & 9,14 \\
\hline$\sigma_{p}^{2}$ & 9,03 & 10,37 & 9,71 & 9,58 & 11,92 & 11,48 \\
\hline$h_{1}^{2}$ & 0,09 & & & 0,17 & & \\
\hline $\mathrm{R}_{\mathrm{g}} / \mathrm{h}_{2}^{2}$ & 0,81 & 0,09 & & 0,98 & 0,12 & \\
\hline $\mathrm{r}_{\mathrm{g}} / \mathrm{h}_{3}^{2}$ & 0,87 & 0,99 & 0,17 & 0,89 & 0,96 & 0,12 \\
\hline
\end{tabular}

$\sigma_{3}^{2}=$ additiv genetische Varianz (auf den Diagonalen), $\operatorname{cov}_{3}=$ additiv genetische Kovarianzen (unter den Diagonalen), $\sigma_{c}^{2}=$ Restvarianz $\sigma_{p}^{2}=$ phănotypische Varianz, $h^{2}=$ Heritabilitat (auf den Diagonalen) und $r_{s}=$ genetische Korrelation (unter den Diagonalen)

Aufgrund der Ergebnisse werden mit einem modifizierten Wiederholbarkeitsmodell (Modell 2) der erste und die weiteren Würfe als getrennte Merkmale betrachtet (Tab. 6). Die Varianzen und die Kovarianz sind bei Linie 04 jeweils größer als bei Linie 03.

Tabelle 6

Genetische Parameter für lebend geborene Ferkel für die Wurfnummern 1 und $\geq 2$ der Linien 03 und 04 (Genetic parameter for live born pigiets for parities 1 and $\geq 2$ for the lines 03 and 04 )

\begin{tabular}{|c|c|c|c|c|}
\hline & \multicolumn{2}{|c|}{ Linie 03} & \multicolumn{2}{|c|}{ Linie 04} \\
\hline & 1 & $\geq 2$ & 1 & $\geq 2$ \\
\hline$\sigma_{a-1}^{2}$ & 1,11 & & 1,66 & \\
\hline $\operatorname{Cov}_{\mathrm{a}} / \sigma_{\mathrm{a}, 22}^{2}$ & 0,91 & 0,97 & 1,35 & 1,36 \\
\hline$\sigma_{m}^{2}$ & 0,12 & - & 0,17 & - \\
\hline$\sigma_{p u}^{2}$ & - & 1,17 & - & 1,48 \\
\hline$\sigma_{c}^{2}$ & 7,72 & 7,71 & 7,79 & 9,04 \\
\hline$\sigma_{p}^{2_{p}^{c}}$ & 8,95 & 9,85 & 9,62 & 11,88 \\
\hline $\mathrm{h}_{1}^{2}$ & 0,12 & & 0,17 & \\
\hline$r_{8} / h_{22}^{2}$ & 0,88 & 0,10 & 0,90 & 0,11 \\
\hline$h_{m}^{2}$ & 0,01 & - & 0,02 & - \\
\hline pu & . & 0,12 & - & 0,12 \\
\hline
\end{tabular}

$\sigma_{\mathrm{a}}^{2}=$ matemale additv genetische Varianz; $\mathrm{h}_{\mathrm{m}}^{2}=$ maternale Heritabilităt 
Bei Linie 04 weist die Wurfgröße beim ersten Wurf einen deutlich höheren $\mathrm{h}^{2}$-Wert im Gegensatz zur wiederholten Wurfleistung der höheren Wurfnummern auf. Ein Vergleich der Tabelle 5 und 6 zeigt, daß bei Linie 03 die additiv genetische Varianz für den ersten Wurf mit dem modifizierten Wiederholbarkeitsmodell höher $\left(\sigma_{\mathrm{a}}^{2}=1,11 \mathrm{zu}\right.$ $0,78)$ und die Restvarianz niedriger $\left(\sigma^{2}=7,72 \mathrm{zu} \mathrm{8,25)}\right.$ geschätzt wird.

Der maternale additiv genetische Effekt liegt bei beiden Linien unterhalb von $2 \%$ an der Gesamtvarianz. Diese relativ niedrigen Werte rechtfertigen nicht die Berücksichtigung des Effektes beim Schätzen von Varianzkomponenten und Zuchtwerten. Eine Ursache dafür ist nicht in der Datenstruktur zu suchen; denn $53 \%$ bzw. $51 \%$ aller Würfe der Linien 03 bzw. 04 gehen auf Vollgeschwisterinformationen zurück. Daher ist der konsequent durchgeführte Wurfausgleich wahrscheinlich die Ursache für unbedeutende maternale genetische Effekte.

Die permanenten Umwelteffekte der wiederholten Würfe weisen bei beiden Linien eine beträchtliche Größenordnung von $\mathrm{pu}=0,12$ auf. Die genetischen Korrelationen zwischen Wurf 1 und den folgenden Würfen liegen bei beiden Linien mit $r_{\mathrm{g}}=0,88$ und 0,90 (Linie 03 und 04) auf einem hohen Niveau. Sie zeigen an, daß bei Verwendung aller Würfe der jeweiligen Sauen in einem Wiederholbarkeitsmodell wahrscheinlich nur eine geringe Verminderung der Genauigkeit gegeben ist.

\section{Genetische Parameter für die Paarungsart}

Bisher sind in der Literatur keine Schätzwerte verfügbar, die den Einfluß der Paarungsart, künstliche Besamung (KB) oder Natursprung (Nat), auf die genetischen $\mathrm{Pa}$ rameter zeigen. Auf dem Vermehrerbetrieb VI werden Sauen der Linie 03 mit Ebern der Linie 04 angepaart. Ungefähr 55\% aller Belegungen erfolgen durch KB. Die Wurfgröße (LGF) liegt bei Verwendung der künstlichen Besamung 0,1 LGF über der Wurfgröße bei Belegung durch Natursprung. Den Einfluß der Belegungsart auf die genetische Beziehung zum Nukleusbetrieb NI zeigt Tabelle 7. Auf dem Nukleusbetrieb werden mehr als $99 \%$ aller Belegungen durch KB vorgenommen. Die Nachkommen von 191 Ebern wurden mit beiden Paarungsarten belegt, so daß für annähernd jeden Eber Informationen zur Verfügung stehen. Nach Belegung durch Natursprung ist die Heritabilität mit $h^{2}=0,08$ niedriger als nach künstlicher Besamung $\left(h^{2}=0,11\right)$.

Tabelle 7

Genetische Parameter der Wurfgröße für unterschiedliche Paarungsarten (KB, Nat) auf dem Vermehrerbetrieb und die genetischen Beziehungen zum Nukleusbetrieb der Linie 03 (Genetic parameter for litter size estimated for different mating types, $\mathrm{AI}$ and Nat, on multiplier farm and the genetic association to nucleus farm of line 03)

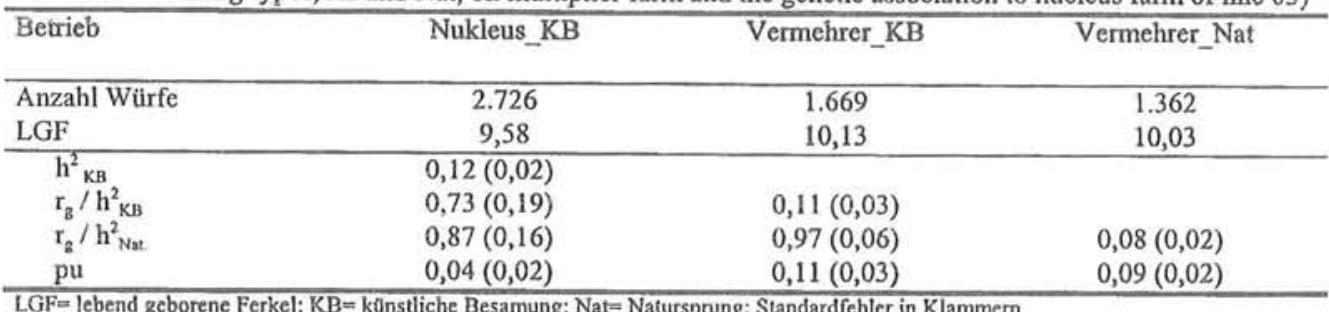

Die genetische Korrelation zwischen den Paarungsarten von $r_{g}=0,97(0,06)$ weist nicht auf genetische Einflüsse auf die Wurfgröße hin. Allerdings werden Unterschiede zwi- 
schen den genetischen Korrelationen zwischen Vermehrer- und Zuchtbetrieb mit $r_{g_{-} K B}=$ $0,73(0,19)$ bzw. $r_{g_{-} \text {Nat }}=0,87(0,16)$ festgestellt.

Die genetische Korrelation von unter 1 ist ein Indiz für Genotyp-Umwelt Interaktionen zwischen Vermehrer- und Nukleusbetrieb. Daher sollen weitere Untersuchungen folgen, in denen genetische Korrelationen zwischen den Zucht- und Vermehrerbetrieben geschätzt werden.

\section{Genotyp-Umwelt-Interaktionen}

Die genetischen Korrelationen zwischen den Betrieben werden mit der Modellgleichung [3] geschätzt. Niedrige Schätzwerte zeigen eine Interaktion zwischen Genotyp und Betriebsumwelt. Die Tabelle 8 zeigt neben den Heritabilitäten für die Wurfgröße auch die genetischen Korrelationen zwischen einzelnen Betrieben von der Nukleus- und Vermehrungsstufe für die Reinzuchtlinien 03 und 04.

Die Heritabilitäten liegen mit $h^{2}=0,12$ und 0,10 auf dem Nukleus NI und dem Vermehrerbetrieb VI deutlich höher als auf dem Nukleus NII mit $h^{2}=0,03$. Auf diesem Betrieb wird ein beträchtlicher Teil der phänotypischen Varianz als permanente Umweltvarianz $(\mathrm{pu}=0,13)$ geschätzt. Die genetische Korrelation von $\mathrm{r}_{\mathrm{g}}=0,76(0,16)$ zwischen NI und VI deutet auf geringe Genotyp-Umwelt-Interaktionen hin. Demgegenüber sind die genetischen Korrelationen zwischen den untersuchten Betrieben und Betrieb NII wesentlich höher. Jedoch sind die Kovarianzen vom Nukleusbetrieb NII durch die niedrige additiv genetische Varianz beeinflußt.

Tabelle 8

Genetische Parameter für lebend geborene Ferkel auf Betrieben der Linien 03 und 04 (Genetic parameter of live bom piglets on farms with lines 03 and 04)

\begin{tabular}{|c|c|c|c|c|c|}
\hline & \multicolumn{3}{|c|}{ Linie 03} & \multicolumn{2}{|c|}{ Linie 04} \\
\hline & $\mathrm{NI}$ & NII & VI & NI & NII \\
\hline Würfe (n) & $\mathrm{n}=2.726$ & $\mathrm{~N}=4.200$ & $\mathrm{n}=3.031$ & $n=2.465$ & $\mathrm{n}=2.580$ \\
\hline$h^{2}$, & $0,12(0,02)$ & & & $0,14(0,03)$ & \\
\hline $\mathrm{r}_{\mathrm{g}} / \mathrm{h}_{\mathrm{z}}^{2}$ & $0,90(0,12)$ & $0,03(0,01)$ & & $0,61(0,36)$ & $0,07(0,02)$ \\
\hline$r_{g} / h^{2}$ & $0,76(0,16)$ & $0,97(0,06)$ & $0,11(0,02)$ & & \\
\hline pu & $0,03(0,02)$ & $0,13(0,01)$ & $0,09(0,02)$ & $0,03(0,03)$ & $0,13(0,02)$ \\
\hline
\end{tabular}

Für die Linie 04 werden die Heritabilitäten für die Nukleusbetriebe NI und NII mit $\mathrm{h}^{2}=$ 0,14 und 0,07 wie schon bei Linie 03 auf unterschiedlichem Niveau angegeben. Beide Schätzwerte sind aber im Vergleich zu Linie 03 höher. Für beide Linien wird auf Betrieb NII ein gleich hoher Wert für die permanente Umwelt geschätzt $(p u=0,13)$. Die genetische Korrelation zwischen den Reinzuchtbetrieben NI und NII liegt mit $r_{\mathrm{g}}=$ $0,61(0,36)$ auf einem mittleren Niveau, was auf Interaktionen zwischen beiden Zuchtbetrieben für Linie 04 hindeutet. Die geringen Wurfzahlen schlagen sich in den Schätzfehlem nieder.

\section{Schlußfolgerungen}

\section{Einflußfaktoren der Wurfgroße}

Die Wurfnummer übt einen bedeutenden Einfluß auf die Wurfgröße aus. Wie die eigene Auswertung (Abb.) und Untersuchungen in der Literatur zeigen (ESTANY und 
SØRENSEN, 1995) können die Wurfnummern 3 bis 5 und die höheren Wurfnummern jeweils zu einer Gruppe zusammengeführt werden, wenn ein Wiederholbarkeitsmodell angewandt wird. Weiterhin kann gezeigt werden, daß die Wurfgröße mit zunehmendem Erstferkelalter linear ansteigt und sich dieser Trend in abgeschwächter Form im zweiten Wurf mit steigender Zwischenwurfzeit fortsetzt. DOBAO et al. (1983) und JØRGENSEN et al. (1989) ermitteln ebenfalls einen linearen Anstieg der Wurfgröße für jeden zusätzlichen Belegemonat. LE COZLER et al. (1998) schließen aus ihren Untersuchungen, daß ein mittleres Erstferkelalter von 356 Tagen für ein gutes Herdenmanagement und effiziente Herdenfruchtbarkeit Vorteile bringt. SCHUKKEN et al. (1994) stellen demgegenüber fest, das frühbelegte Sauen einen stärkeren Zuwachs in der Wurfgröße des zweiten Wurfes haben und somit einen Teil des Rückstandes gegenüber später belegten Sauen kompensieren. Unter Berücksichtigung von Wurfgröße und Lebensdauer einer Sau ist daher der Gewinn nicht signifikant vom Alter bei der ersten Konzeption beeinflußt (SCHUKKEN et al., 1994).

Eine mittlere Zwischenwurfzeit von 139 bis 158 Tagen erweist sich ab dem dritten Wurf als optimal für die Wurfgröße. Problemsauen, die in kleineren oder größeren Wurfintervallen abferkeln, haben tendentiell kleinere Würfe.

HOFER (1998) und THOLEN et al. (1998) berücksichtigen den Wurfabstand trotz niedriger Heritabilität (HALEY et al., 1988) und geringem ökonomischen Gewicht (KRIETER, 1994) als weiteres Merkmal. TEN NAPEL et al. (1992 und 1995) schätzen eine Heritabilität für das Intervall vom Absetzen bis zum Östrus auf $h^{2}=0,17$. Sie zeigen, daß die Selektion das untersuchte Intervall durch eine Reduzierung der Sauen mit höheren Intervallen verkürzt. Daher sollte in folgenden Untersuchungen das Intervall Absetzen bis zum Östrus bzw. der Anteil Sauen, die bis zum sechsten Tag brünstig werden, berücksichtigt werden. Dieser Zeitraum ist maßgeblich für die Variation des Wurfabstandes verantwortlich und ein leicht zu erfassender Erfolgsparameter für die Fruchtbarkeit einer Sau und einer Herde.

Herde-Jahres-Saison Effekte sind ein weiterer bedeutender Einflußfaktor. In einigen Datensätzen sind die Klassen bedingt durch kleine Betriebsstrukturen gering besetzt (ESTANY und SØRENSEN, 1995; MERKS und MOLENDIJK, 1995; FREY et al., 1995). FREY et al. (1995) zeigen mit einem Vergleich von Modellen mit fixen und zufalligen Betriebsjahren, daß bei kleinen Zeitgefährtengruppen mit weniger als 10 Beobachtungen je Betrieb-Jahr-Subklasse das Modell mit zufälligen Herde-Jahr Effekten zu einer genaueren Vorhersage führt. Neben der Zahl der Beobachtungen je Subzelle und Beziehung zwischen Vater und Zeitgefährtengruppe haben die Datenstruktur und das Zuchtsystem einen Einfluß auf die Entscheidung, ob Zeitgefährtengruppen als zufällige oder fixe Effekte berücksichtigt werden (BRADFIELD, 1998). In der vorliegenden Untersuchung war jedoch eine ausreichende Klassenbesetzung vorhanden, so daß Verzerrungen der Schätzwerte weitgehend ausgeschlossen werden können.

\section{Genetische Parameter der Wurfgröße}

Die genetische Korrelation zwischen dem ersten und zweiten bzw. den folgenden Würfen entscheidet darüber, ob ein (modifiziertes) Wiederholbarkeits- oder ein Mehrmerk- 
malsmodell bei der genetischen Analyse zur Anwendung kommt.

Diese Untersuchung bestätigt, daß die genetischen Korrelationen zwischen erstem und dritten bzw. zweitem und dritten Wurf mit $r_{g}=0,87$ bis 0,99 auf hohem Niveau liegen (Tab. 5). Der dritte Wurf muß daher nicht getrennt vom zweiten Wurf berücksichtigt werden. Bei bivariaten Schätzungen mit zusammengefaßten Würfen $(\geq 2)$ werden die genetischen Korrelationen für die Linien 03 und 04 mit $r_{g}>0,80$ ebenfalls auf ein hohes Niveau geschätzt (Tab. 6). Der geringe Anteil der maternalen additiv genetischen Varianz an der Gesamtvarianz, der wahrscheinlich auf konsequenten Ausgleich der Wurfgröße zurückzuführen ist, rechtfertigt nicht die Berücksichtigung im Schätzmodell. Daher wird für beide Reinzuchtlinien ein Wiederholbarkeitsmodell empfohlen, mit dem permanente Umwelteffekte für die wiederholten Wurfleistungen geschätzt werden. Ein weiterer Grund für bivariate Schätzungen können unterschiedliche Heritabilitäten für einzelne Würfe sein, wie sie bei Linie 04 auftreten. Da langfristige Eliminierung von maternalen Effekten der Wurfleistung durch Umsetzen von Ferkeln zu einer Verminderung des Niveaus der mütterlichen Eigenschaften (intrauterine Ernährung, Milchleistung, etc.) führen kann, sind Merkmale, die diese Eigenschaften verbessern wie maternale Effekte des Ferkelgeburts- und des Ferkelabsetzgewicht um so bedeutender unter Bedingungen des Wurfgrößenausgleichs (RÖHE, 1998).

Die Paarungsart übt einen Einfluß auf die genetische Beziehung zwischen Nukleusund Vermehrerbetrieb aus $\left(r_{g_{\_} N a t}=0,87, r_{g_{K} K_{B}}=0,73\right)$. Beim Natursprung ist die genetische Korrelation höher, jedoch die Heritabilität mit $h^{2}{ }_{\text {Nat }}=0,08$ gegenüber $h^{2}{ }_{K B}=0,11$ niedriger (Tab. 7). Die Parameterschätzungen auf einzelnen Betrieben zeigen deutliche Unterschiede bei den Heritabilitäten (Tab. 8). Auf dem Betrieb NII sind bei beiden Linien niedrige $\mathrm{h}^{2}$-Werte mit sehr hohen Schätzwerten für die permanente Umwelt $(\mathrm{pu}=$ $0,13)$ gepaart. Zwischen den Betrieben NI und NII der Linie $04\left(r_{g}=0,61\right)$, wie auch zwischen NI und VI der Linie $03\left(r_{g}=0,76\right)$, werden geringfügige Genotyp-UmweltInteraktionen festgestellt. Diese Werte lassen aber keine gravierenden Rangverschiebungen bei der Selektion von Ebern erwarten.

\section{Literatur}

ALFONSO, L.; NOGUERA, J.L.; BABOT, D.; ESTANY, J.:

Estimates of genetic parameters for litter size at different parities in pigs. Livest. Prod. Sci. 47 (1997), 149-156

ANDERSEN, S.:

Status report- The National Danish Pig Breeding Program. Internation. Workshop "Introduction of BLUP Animal Model in Pigs", Prag-Uhrineves (1998)

BIDANEL, J.P.; DUCOS, A.; GROENEVELD, E.; GRUAND, J.; LAGANT, H.; LEGAULT, C.: Genetic variability of components of litter size in French Large White gilts. 43th Annual Meeting EAAP, Madrid, Spain (1992)

BOLDMAN, K.G.; KRIESE, L.A.; VAN VLECK, L.D; KACHMAN, S.D.:

A Manual for use of MTDFREML. A set of programs to obtain estimates of variances and covariances. U.S. Department of Agricultural Research Service (1993)

BÖSCH, M.; RÖHE, R.; LOOFT, H.; KALM, E.:

Schătzung der genetischen Beziehungen zwischen der Reinzucht- und Kreuzungsleistungen für die Fruchtbarkeit beim Schwein. Vortragstagung der DGrZ/GrT (1997), Bonn

BRADFIELD, M:

personal communication (1998) 
BRISBANE, J.; SULLIVAN, B.; CHESNAIS, J.:

Status report: The Canadian Swine Improvement Program (CSIP). Internat. Workshop "Introduction of

BUNTER, K.L.: BLUP Animal Model in Pigs", Prag-Uhrineves, (1998)

Genetic relation between age at first farrowing, sow stayability, and other sow reproductive traits. Proc. $12^{\text {th }}$ Confer. AAABG, Dubbo, NSW, Australia: Part 1. (1997), 503-511

DOBAO, M.T.; RODRIGANEZ, J.; SILLIO, L.:

Seasonal influence on fecundity and litter performance characteristics in Tberian pigs. Livest. Prod. Sci. 10 (1983), 601-610

ESTANY, J.; SØRENSEN, D.:

Estimation of genetic parameters for litter size in Danish Landrace and Yorkshire pigs. Anim. Sci. 60 (1995), 315-324

FALCONER, D.S.:

Introduction to Quantitative Genetics. Fourth Edition (1996), Longman Group UK

FERRAZ, J.B.S.; JOHNSON; R.K.:

Animal model estimation of genetic parameters and response to selection for litter size and weight, growth and backfat in closed seedstock populations of Large White and Landrace swine. J. Anim. Sci.

FISCHER, R.:

Schätzung genetischer Parameter für Reinzucht- und Kreuzungsleistungen beim Schwein. Univ. HalleWittenberg, Diss., 1998

FREY, M.; HOFER, A.; KƯNZI, N.:

Zuchtwertschätzung für die Wurfgröße beim Schwein. Vortragstagung der DGfZ/GfT 1994, Halle

FREY, M.; HOFER, A.; KÜNZI, N.:

Vergleich von Modellen mit fixem oder zufälligem Herde*Zeitperioden-Effekt. Vortragstagung der DGfZ/GfT 1995, Hannover

GRANDJOT, G.; BRANDT, H.; GLODEK, P.:

Genetische und phänotypische Untersuchungen zu Eigenleistungs-, Sperma- und Fruchtbarkeitsmerkmalen von Besamungsebern. 1. Mitteilung: Systematische Einflußfaktoren und GROENEVELD, E.:

VCE4 User's Guide and Reference Manual Version 1.1. (1998)

HALEY, C.S.; AVALOS, E.; SMITH, C.:

Selection for litter size in the pig. Anim. Breed. Abstr. 56 (1988), 317-332

HALEY, C.S.; LEE, G.J.:

Genetic factors contributing to variation in litter size in British Large White gilts. Livest. Prod. Sci. 30 (1992), 99-113

HERMESCH, S.:

Genetic parameters for lean meat yield, meat quality, reproduction and feed efficiency traits for HOFER, A.: Australian pigs. PhD dissertation. Dep. of Animal Science, Univ. of New England, Australia (1996)

Status Report: Genetic evaluation in the Swiss National Breeding Program. Internat. Workshop "Introduction of BLUP Animal Model in Pigs", Prag-Uhrineves (1998)

IRGANG, R.; FAVERO, J.A.; KENNEDY, B.W.:

Genetic parameters for litter size of different parities in Duroc, Landrace and Large White sows. J. Anim. Sci. 72 (1994), 2237-2246

JøRGENSEN, J.N.:

KRIETER, J.:

The influence of maternal effects on litter size in pigs. Acta Agric. Scand. 39 (1989), 421-429

Zuchtplanung beim Schwein. Habilitationsschrift. Schriftenreihe des Instituts für Tierzucht und Tierhaltung an der CAU Kiel; Heft 81 (1994)

LAMBERSON, W.R.; JOHNSON, R.K.; ZIMMERMAN, D.R.; LONG, T.E.:

Direct responses to selection for increased litter size, decreased age at puberty or random selection following selection for ovulation rate in swine. J. Anim. Sci. 69 (1991), 3129-3143 
LE COZLER, Y.; DAGORN, J.; LINDBERG, J.E.; AUMAITRE, A.; DOURMAD, J.Y.:

Effect of age at first farrowing and herd management on long-term productivity of sows. Livest. Prod. LOOFT, H.: Sci. 53 (1998), 135-142

Analyse der Hyperprolific-Selektion und Schätzung von Varianzkomponenten für Fruchtbarkeitsmerkmale beim Schwein. Diss., Schriftenreihe des Instituts für Tierzucht und Tierhaltung an der CAU Kiel; Heft 67 (1992)

MERKS, J.W.M.:

Genotype*Environment Interactions in pig breeding programmes. $\mathrm{PhD}$. Thesis, Wageningen (1988)

MERKS, J.W.M.; MOLENDIJK, R.J.F.:

Genetic correlations between production traits and first parity traits. $46^{\text {th }}$ Annual Meeting EAAP, Prag (1995)

OMTVEDT, I.T.; STANISLAW, C.M.; WHATLEY, J.A. Jr.:

Relationship of gestation length, age and weight at breeding and gestation gain to sow productivity at farrowing. J. Anim. Sci. 24 (1965), 531-535

RÖHE, R.; KENNEDY, B.W.:

The influence of maternal effects on accuracy of evaluation of litter size in swine. J. Anim. Sci. 71 (1993), 2353-2364

RÖHE, R.; KENNEDY, B.W.:

Estimation of genetic parameters for litter size in Canadian Yorkshire and Landrace swine with each

RÖHE, R.: parity of farrowing treated as a different trait. J. Anim. Sci. 73 (1995), 2959-2970

Genetic statistical analysis of reproduction, neonatal survival and birth weight in order to optimize selection for sow productivity. Univ. Kiel, Habilitationsschrift 1998

RYDHMER, L.; ELIASSON-SELLING, L.; JOHANSON, K.; STERN, S.; ANDERSSON,S.:

A genetic study of estrus symptoms at puberty and their relationship to growth and leanness in gilts. J. Anim. Sci. 72 (1994), 1964-1970

SAS INSTITUTE INC.:

SAS/STAT User's Guide, Version 6, 4. Edition, Vol. 1 and 2, 1995

SEE, M.T.; MABRY, J.W.; BERTRAND, J.K.:

Restricted maximum likelihood estimation of variance components from field data for number of pigs born alive. J. Anim. Sci. 71 (1993), 2905-2909

SCHUKKEN, Y.H.; BUURMAM, J.; HUIRNE, R.B.M.; WILLEMSE, A.H.; VERNOY, J.C.M.; VAN DEN

BROEK, J.; VERHEIJDEN, J.H.M.:

Evaluation of the optimal age at first conception in gilts from data collected in commercial swine herds. J. Anim. Sci. 72 (1994), 1387-1392

SØRENSEN, D.A.:

An animal model for selection for litter size in the Danish pig breeding program. 4th WCGALP, Edinburgh, Vol. 15 (1990), 435-438

SOUTHWOOD, O.I.; KENNEDY, B.W.:

Estimation of direct maternal genetic variance for litter size in Canadian Yorkshire and Landrace swine using an animal model. J. Anim. Sci. 68 (1990), 1841-1847

TÄUBERT, H.; BRANDT, H.; GLODEK, P.: Correlation between purebred and crossbred performance for litter traits in swine. $48^{\text {th }}$ Annual Meeting

TÄUBERT, H.: EAAP, Vienna, G6.2 (1997)

Schälzung von Varianz- und Kovarianzkomponenten für die Wurfgröße von Reinzucht- und Kreuzungssauen. Diss., Göttingen (1998)

TEN NAPEL, J.; ARGYROPOULOS, S.; DE VRIES, A.G.; TE BRAKE, J.H.A.:

Direct and correlated responses of selection on interval weaning oestrus in pigs. 43rd Annual Meeting EAAP, Madrid, Spain (1992)

TEN NAPEL, J.; DE VRIES, A.G.; BUITING, G.A.J.; LUITING, P.; MERKS, J.W.M.; BRASCAMP, E.W.: Genetics of the interval from weaning to estrus in first-litter sows: Distribution of data, direct response of selection, and heritability. J. Anim. Sci., 73 (1995), 2193-2203 
THOLEN, E.; BUNTER, K.L.; HERMESCH, S.; GRASER, H.U.:

The genetic foundation and reproduction traits in Australian pig populations. 2. Relationship between weaning to conception interval, reproduction and production traits. Aust. J. Agric. Res. 47 (1996), 1275-1290

THOLEN, E., STORK, F.J.; TRAPPMANN, W.; SCHELLANDER, K.:

Status Report: Herdbook Pig Breeding Societies in North-Rhine-Westfalia. Internat. Workshop "Introduction of BLUP Animal Model in Pigs", Prag-Uhrineves (1998)

TÖLLE, K.-H.; TRAPPMANN, W.:

Ansätze zur Zuchtwertschätzung für Reproduktionsmerkmale beim Schwein. Vortragstagung der DGfZ/GfT, Hannover, (1995)

TRIBOUT, T.; MAIGNEL, L.; BIDANEL, J.P.:

National genetic evaluation of pigs in France. Internat. Workshop "Introduction of BLUP Animal Model in Pigs", Prag-Uhrineves (1998)

VON FELDE, A.:

Genetische Analyse der Futteraufnahme-Informationen von Jungebern aus Gruppenprüfung mit automatischen Fütterungsanlagen. Schriftenreihe des Instituts für Tierzucht und Tierhaltung an der CAU Kiel; Heft 90 (1996)

WILLEKE, H.; RICHTER, L.:

Der Einfluß des Ebers auf die Wurfgröße beim Schwein. Genetisch-statistische Auswertungen von Fruchtbarkeitsdaten einer Besamungspopulation. Züchtungskunde, Stuttgart 52 (1980), 438-443

WOLF, J.; GROENEVELD, E.; WOLFOVA, M.; JELINKOVA, V.; PRAZAK, C.:

Genetic evaluation of pigs in Czech Republic- Status Report. Internat. Workshop "Introduction of BLUP Animal Model in Pigs", Prag-Uhrineves (1998)

Eingegangen: 03.08 .1999

Akzeptiert: 30.09 .1999

Anschriften der Verfasser

Dr. MARKUS BÖSCH, PD Dr. RAINER RÖHE, Prof. Dr. Dr. h.c. mult. ERNST KALM

Christian-Albrechts-Universităt zu Kiel

Institut für Tierzucht und Tierhaltung

Olshausenstr. 40

D-24098 Kiel

Dr. HOLGER LOOFT

PIC Deutschland GmbH

Ratsteich 31

D-24837 Schleswig 